\title{
Mesenchymal hepatic hamartoma associated with elevated alpha fetoprotein mimicking a hepatoblastoma: A rare case and a literature review
}

\author{
Luvo Gaxa, Bafana Hlatshwayo
}

\begin{abstract}
Introduction: A mesenchymal hepatic hamartoma is a heterogeneous and a rare congenital benign neoplasm whose presentation may be cystic, solid or mixed and the mass may demonstrate varying degrees of vascularity. Treating mesenchymal hepatic hamartomas conservatively is not encouraged because these lesions have the potential to grow rapidly and may degenerate to a malignant undifferentiated embryonal sarcoma. The standard treatment of mesenchymal hepatic hamartoma is surgical resection. However, marsupialization is also indicated in surgically unresectable cases. Case Report: We report a case of one-year-old girl who presented with a one-month history of abdominal distention. The patient was born through a normal vaginal delivery at term. Apgar score 9/10-10/10, birth weight $2.8 \mathrm{~kg}$. Head circumference $36 \mathrm{~cm}$. The patient is retro-viral disease negative. She has normal developmental milestones and the immunizations are up to date. Ultrasound of the abdomen was performed and it showed right liver lobe large masses. Computed tomography (CT) scan was subsequently performed to further characterize the liver lesions. Ultrasound guided biopsy was
\end{abstract}

Luvo Gaxa1, Bafana Hlatshwayo ${ }^{2}$

Affiliations: ${ }^{1} \mathrm{MBChB}$, Mmed (Rad D,)Senior Specialist, Paediatric Radiology Fellow, South Africa; ${ }^{2}$ Dip Rad Diag, MBChB, MMed Rad D, Senior Specialist, Diagnostic Radiology and Imaging, Polokwane-Mankweng Hospital Complex, Polokwane, Limpopo, South Africa.

Corresponding Author: Luvo Gaxa, MBChB, Mmed (Rad D) Senior Specialist,Paediatric Radiology Fellow, South Africa. Email: luvogaxa@yahoo.com

Received: 23 April 2016

Accepted: 13 September 2016

Published: 30 March 2017 performed and it confirmed the diagnosis of mesenchymal hepatic hamartoma. The alphafetoprotein was markedly elevated. Conclusion: It is of utmost importance to note that as much as the mesenchymal hepatic hamartoma typically shows a normal alpha-fetoprotein, a rare presentation of this benign tumor may be associated with markedly elevated alpha fetoprotein.

Keywords: Mesenchymal hepatic hamartoma, Rare, Treatment

\section{How to cite this article}

Gaxa L, Hltshwayo B. Mesenchymal hepatic hamartoma associated with elevated alpha fetoprotein mimicking a hepatoblastoma: A rare case and a literature review. Case Rep Int 2017;6:9-12.

Article ID: 100034CRINTLG2017

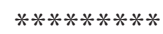

doi:10.5348/crint-2017-34-CR-3

\section{INTRODUCTION}

Mesenchymal hamartoma of the liver is defined as a benign tumor of infancy with features of epithelial structures and mixture in a loose connective tissue stroma suggestive of lymphangiomatous channels with fluid accumulation [1].

Doner et al., Parkard and Palmer state that hepatic hamartoma is a portal connective tissue developmental anomaly during fetal life as opposed to a true neoplasm [1].

Etiology: Edmondson speculated that hepatic hamartoma might be the consequence of failure of 
embryonic fetal liver's normal development or a degenerative accessory lobe change [1].

Mesenchymal hepatic hamartoma may also be defined as a developmental anomaly that is rare and benign characterized by mixture of loose mesenchymal tissue, connective tissue, bile ducts and hepatocytes along with cysts formed from degenerative areas of mesenchyme [2].

Incidence: The clinical presentation of mesenchymal hepatic hamartoma is common in the first 24 months of life and the median age of occurrence is 10 months [3].

Males are commonly affected compared to females at a ratio of 2:1 and there is preponderance of the right liver lobe compared to the left liver lobe [4].

Ever since the first cases of mesenchymal hepatic hamartoma were reported in year 1959 and 1966, as of year 2012 less than 200 cases have been reported in English literature [2].

Characteristically, the laboratory findings show normal liver function tests although few reports of elevated alpha fetoprotein of more than $6000 \mathrm{ng} / \mathrm{ml}$ are documented in literature [3].

\section{CASE REPORT}

A one-year-old girl presents with a one-month history of abdominal distention. No previous medical history reported. The patient was born through a normal vaginal delivery at term. Apgar score 9/10-10/10, birth weight $0.8 \mathrm{~kg}$. Head circumference $36 \mathrm{~cm}$. The patient is retro-viral disease negative. She has normal developmental milestones and the immunizations are up to date. Ultrasound of the abdomen was performed and it showed right liver lobe large masses. Computed tomography (CT) scan was subsequently performed to further characterize the liver lesions. Computed tomography scan revealed that: there was a large liver mass, heterogeneously enhancing post contrast and the mass also demonstrated cystic changes within it. The mass was noted to cross the midline to the contra-lateral side and displacing the abdominal organs inferolaterally. The differential diagnosis given were 1. hepatoblastoma, and 2. hepatocellular carcinoma.

The alpha fetoprotein was markedly elevated measuring $3866 \mathrm{ng} / \mathrm{ml}$. White cell count was 21, erythrocyte sedimentation rate 10 , hemoglobin 8.8 . The temperature $36.3^{\circ} \mathrm{C}$, pulse rate 124 beats/min, saturation $100 \%$ on room air. Biopsy was ultrasound guided and it was repeated twice to confirm that the diagnosis was indeed a hepatic hamartoma as opposed to hepatoblastoma.

Microscopy sections demonstrate cores of lesional tissue. The lesion under low power demonstrated a fibroadenoma-like architectural appearance. The lesion comprises predominance of mature fibroblastic proliferation appearing within a fibromyxoid background. There is accompanying cystically dilated bile ducts some of which demonstrate evidence of proliferation. In parts, entrapped trabeculae and cords of resident liver tissue are present. This is arranged haphazardly. There is no evidence of malignant transformation. Some of the hepatocytes contain bile pigment. In addition to chronic inflammatory cells, scattered eosinophils are present. There is no evidence of malignancy. The features are those of a mesenchymal hamartoma of the liver.

The patient was transfused $130 \mathrm{ml}$ of red packed cells four hourly. Lasyx $9 \mathrm{mg}$ intravenously was given post transfusion. Zinc sulfate $10 \mathrm{mg}$ per os daily, ondansetron hydrochloride and the patient was given $2 \mathrm{mg}$

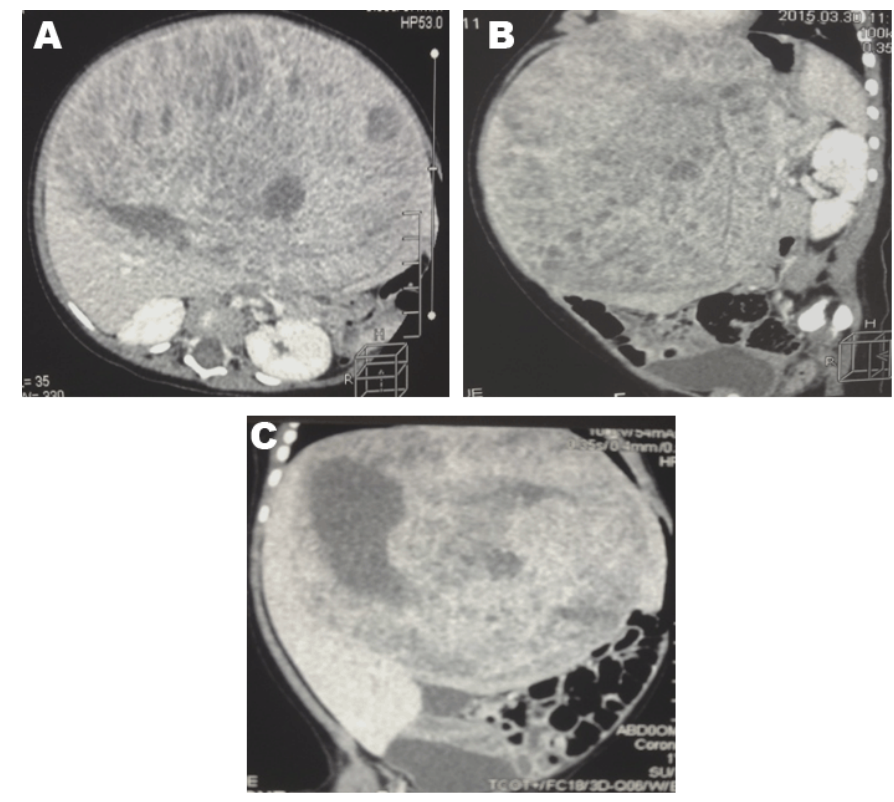

Figure 1: (A) Axial view post contrast computed tomography scan of the liver showing a large heterogeneously enhancing liver mass with cystic changes within it. (B, C) Coronal post contrast computed tomography scan of the liver confirm the large liver mass crossing the midline to the contra-lateral side and displacing the bowel and the abdominal organs inferolaterally.

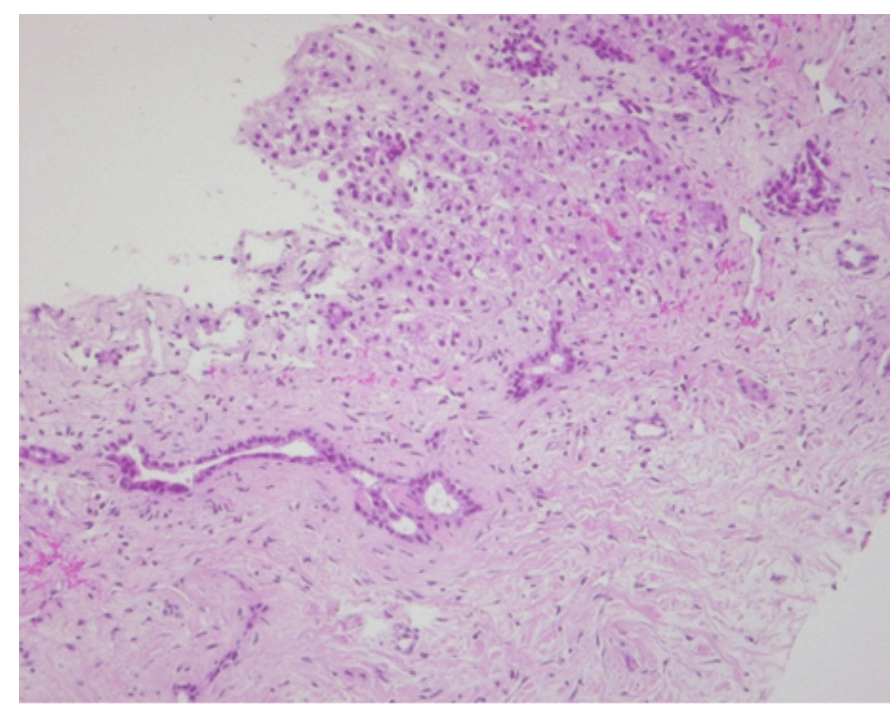

Figure 2: Histopathology showing lesion comprising of predominance of mature fibroblastic proliferation appearing within a fibromyxoid background (H\&E stain, x100). 
intravenously 12 hourly and zyloprin $100 \mathrm{mg}$ per os three times a day were given because she presented with severe anemia and diarrhea and vomiting due to gastroenteritis.

The patient is being discussed with the pediatric surgeons for further management.

\section{DISCUSSION}

Mesenchymal hamartoma of the liver is defined as a benign tumor of infancy with features of epithelial structures and mixture in a loose connective tissue stroma suggestive of lymphangiomatous channels with fluid accumulation [1]. Edmondson speculated that hepatic hamartoma might be the consequence of failure of embryonic fetal liver normal development or a degenerative accessory lobe change [1].

Mesenchymal hepatic hamartoma may also be defined as a developmental anomaly that is rare and benign characterized by mixture of loose mesenchymal tissue, connective tissue, bile ducts and hepatocytes along with cysts formed from degenerative areas of mesenchyme [2]

A mesenchymal hepatic hamartoma is a heterogeneous and a rare congenital benign neoplasm whose presentation may be cystic, solid or mixed and the mass may demonstrate varying degrees of vascularity [3].

This primary benign liver tumor mainly occurs during infancy and childhood. However; a handful of cases have been reported in older age groups as well. The first cases of mesenchymal hepatic hamartoma were reported in year 1959 and 1966 and as of year 2012 less than 200 cases have been reported in English literature ever since year 1956. Some of the names that are used for mesenchymal hepatic hamartoma are cystic hamartoma, pseudocystic mesenchymal tumors, lymphangiomas, bile duct hamartomas and mesenchymomas [4].

It is estimated that $55 \%$ of mesenchymal hepatic hamartomas are diagnosed in the first year of life and $75 \%$ of the cases occur in the right liver lobe and the remainder of the cases occur in the left liver lobe or involve both lobes. At its early stages the mesenchymal hepatic hamartoma is asymptomatic and may be diagnosed co-incidentally on imaging or physical examination. However, the clinical presentation is that of an upper quadrant mass and or abdominal distention [5].

The appearance of a mesenchymal hepatic hamartoma at imaging largely depends on its gross pathologic appearance and may range from a cystic mass that has either thin or thick septations to a predominantly solid mass which contains a few cystic areas. At ultrasound the cystic type of mesenchymal hepatic hamartoma presents as an anechoic or nearly anechoic mass with echogenic thin or thick septations and the solid type presents as an echogenic mass. The gelatinous components of the mass may show low-level echoes within the cystic mass and on color Doppler ultrasound there is minimal blood flow that is only limited to the septa and the solid component [6].
Radiographic findings may demonstrate a calcification within the hepatic tumor although this is not the common finding. On computed tomography scan, the mass is commonly hypo-dense and on post contrast examination the septa enhances. T2-weighted on magnetic resonance imaging (MRI): image may show low or high signal intensity masses [5].

The solid type of mesenchymal hepatic hamartoma presents as a low signal mass on both T1-weighted and T2-weighted images secondary to fibrosis and the septa enhance following gadolinium studies [5, 7].

Laboratory findings typically show normal liver function tests although reports of elevated alpha fetoprotein of more than $6000 \mathrm{ng} / \mathrm{ml}$ are documented in literature [5].

If possible, complete surgical resection with hepatic lobectomy or non-anatomic excision is the definitive treatment. For unresectable lesions, drainage with marsupialization and partial resection may be performed although this may be followed by recurrence of the mass. Treating mesenchymal hepatic hamartomas conservatively is not encouraged because these lesions have the potential to grow rapidly and may degenerate to a malignant undifferentiated embryonal sarcoma [7].

The differential diagnosis of mesenchymal hepatic hamartoma include hepatic abscess, Caroli's disease, metastasis, hepatoblastoma, undifferentiated embryo sarcoma of the liver, hemangioma, hepatic hydatid disease and infantile hemangioendothelioma [8, 9].

\section{CONCLUSION}

A mesenchymal hepatic hamartoma associated with elevated alpha fetoprotein is a rare and an exceptional encounter. The top differential diagnosis of this rare presentation is a hepatoblastoma.

$* * * * * * * * *$

\section{Acknowledgements}

We are thankful to our Radiographers Molopa Moyahabo Tink, Makuka Emilly Mogashoa, Sina Aphane, Pontso Male and Karina Stander for retrieving and preparing images for this case study.

\section{Author Contributions}

Luvo Gaxa - Substantial contributions to conception and design, Acquisition of data, Analysis and interpretation of data,drafting the article, Revising it critically for important intellectual content, Final approval of the version to be published

Bafana Elliot Hlatshwayo - Substantial contributions to conception and design, Revising it critically for important intellectual content, Final approval of the version to be published 


\section{Guarantor}

The corresponding author is the guarantor of submission.

\section{Conflict of Interest}

Authors declare no conflict of interest.

\section{Copyright}

(C) 2017 Luvo Gaxa et al. This article is distributed under the terms of Creative Commons Attribution License which permits unrestricted use, distribution and reproduction in any medium provided the original author(s) and original publisher are properly credited. Please see the copyright policy on the journal website for more information.

\section{REFERENCES}

1. Gupta R, Parelkar SV, Sanghvi B. Mesenhymal harmatoma of the liver. Indian J Med Paedatr Oncol 2009 Oct-Dec;30(4):141-3.

2. Anil G, Fortier M, Low Y. Cystic hepatic mesenchymal hamartoma: the role of radiology in diagnosis and perioperative management. $\mathrm{Br} \mathrm{J}$ Radiol 2011 May;84(1001):e91-4.
3. Atas E, Demirkaya M, Kesik V, Balamtekin N, Kocaoglu M. Mesenchymal hamartoma of the liver mimicking hydatid cyst. Pediatr Therapeut 2012;2:3.

4. Majidpour HS, Yousefine V, Salehi MG. Hepatic Mesenchymal hamartoma. Pak J Med Sci 2008 OctDec;24(6):876-8.

5. Chung EM, Cube R, Lewis RB, Conran RM. From the archives of the AFIP: Pediatric liver masses: Radiologic-pathologic correlation part 1. Benign tumors. Radiographics 2010 May;30(3):801-26.

6. Markhardt BK, Rubens DJ, Huang J, Dogra VS. Sonographic features of biliary hamartomas with histopathologic correlation. J Ultrasound Med 2006;25:1631-3.

7. Siddiqui MA, McKenna BJ. Hepatic mesenchymal hamartoma: A short review. Arch Pathol Lab Med 2006 Oct;130(10):1567-9.

8. Chandramouleeswari K, Anita S, Shivali B. Mesenchymal hamartoma of the liver: A case report. J Clin Diagn Res 2012 Nov;6(9):1552-4.

9. Ye $\mathrm{BB}, \mathrm{Hu} \mathrm{B}$, Wang LJ, et al. Mesenchymal hamartoma of liver: Magnetic resonance imaging and histopathologic correlation. World J Gastroenterol 2005 Oct 7;11(37):5807-10.
Access full text article on other devices

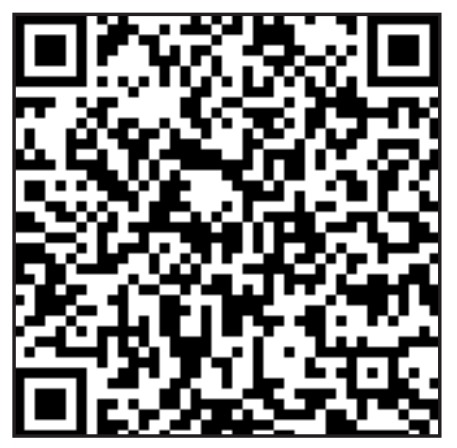

Access PDF of article on other devices

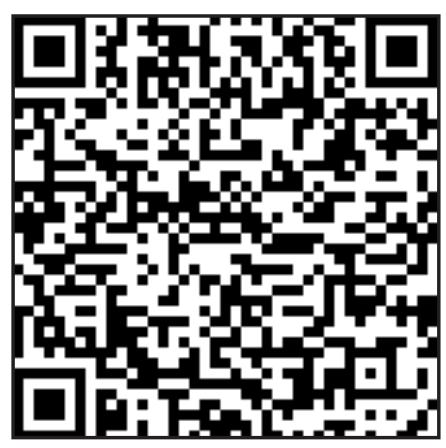

